\title{
Extraction and Feature Analysis of Mouse Trabecular with Active Contour Model Based on Micro-CT Images
}

\author{
Shu-yue $\mathrm{CHEN}^{*}$, Ying LI and Kai-bin CHU \\ School of Information Science and Engineering, Changzhou University, Changzhou \\ 213164, Jiangsu Province, China \\ *csyue2000@163.com
}

\begin{abstract}
Keywords: Micro-CT, Mouse Trabecular, LGIF Model, K-Means Cluster, Active Contour, Image Feature Analysis.
\end{abstract}

\begin{abstract}
To solve the non-uniformity of micro-CT image with CV(Chan-Vese) model and the influence of location of initial contour curves on segmentation speed in the LGIF(Local and Global Intensity Fitting) model, K-LGIF(K-means-Local and Global Intensity Fitting) model was proposed through adding K-means clustering information into energy function of LGIF active contour model. The K-LGIF model extracts outline of the image as the initial contour to reduce the number of iterations and shorten time consuming. Comparing measured geometry parameters by simulating symptoms of osteoporosis and normal mouse femur of trabecular bone and using gray level co-occurrence matrix, we measured the parameters of texture distribution of trabecular bone. The experimental results show that the K-LGIF model can effectively improve segmentation of non-uniform gray image and increase speed of segmentation. This method may provide an approach for the quantitative analysis of osteoporosis.
\end{abstract}

\section{Introduction}

Micro-CT (micro computed tomography) is called microscopic CT[1]. This technology has the ability to scan for live animals or a variety of hard tissue and relevant soft tissue in the body and analyze the 3D model platform. It is of good "microscopic" effect. The advantage of this technology is that without destroying the sample to learn more about the microstructure and characteristic parameters of the internal sample.

Mumford and Shah functional model[2] takes advantage of energy minimization process to solve image segmentation problem. But because the local into minimum value is the one big drawback of this model, it is more difficult in the solution process. Chan and Vese simplified Mumford Shah model through implicit established a piecewise constant CV model[3] of two phase image segmentation. The model can be divided by the image as a background and target, which used the differences from the average gray value of target and background and changing automatically the topology of the curve in the evolution process for image segmentation. This method can effectively segment the uniform strength and little noise image. But at the same time, the uneven gray scale of medical images in image segmentation using CV model is not obvious. Li Chunming[4-5] who proposed to refer to the point of the Gaussian kernel function, and the function as a local binary kernel function to fit the energy of local binary fitting model (LBF), solved the shortcomings of the CV model that contains the image of local information. Li Wang put forward LGIF model which combined with the advantages of the CV model and LBF model[6]. It is global regional information and local area information geometric active contour model. Meanwhile, the low contrast image segmentation and noise robustness problem has been greatly improved with the 
model.

Considering the speed issue of segmentation with LGIF model, using the K-means clustering [7-8] for obtaining image information and getting preliminarily initial active contour line of the model to reduce the number of iterations and accelerate the convergence rate of the active contour before image segmentation. Therefore, the segmentation efficiency of the model is improved.

\section{K-LGIF active contour extraction model}

The LGIF model combined with the advantages of the CV model and LBF model, and not only segmented effectively the low contrast images but reduced the impact of noise on the image segmentation. However, the initial contour $C$ is a regular graph in general models, for example: rectangular, triangular, etc. It improved the speed of level set evolution. In this paper, the above shortcomings, K-means clustering information is put forward to join the LGIF model, the experiment proved that the improved model can accelerate effectively the speed of active contour evolution.

To avoid active contour line initialization, the penalty term is added in the LGIF model, given the level set function in level set method: $\Omega \rightarrow R$, point $x \in \Omega$, contour line $C \in R$. The energy functional is defined:

$$
\begin{aligned}
& E\left(c_{1}, c_{2} f_{1}, f_{2}, \phi\right)=(1-\omega)\left\{\lambda_{1} \int_{\Omega}\left[\int_{\text {outside }(c)} K_{\sigma}(x-y)\left|I(y)-f_{1}(x)\right|^{2} H_{\varepsilon}(\phi(y)) d y\right] d x+\right. \\
&\left.\lambda_{2} \int_{\Omega}\left[\int_{\text {inside }(c)} K_{\sigma}(x-y)\left|I(y)-f_{2}(x)\right|^{2}\left(1-H_{\varepsilon}(\phi(y))\right) d y\right] d x\right\}+ \\
& \omega\left[\lambda_{1} \int_{\text {outside }(c)} F .\left|I(x)-c_{1}\right|^{2} H_{\varepsilon}(\phi(x)) d x+\right. \\
&\left.\lambda_{2} \int_{\text {inside }(c)} F .\left|I(x)-c_{2}\right|^{2}\left(1-H_{\varepsilon}(\phi(x))\right) d x\right]+\mu|\phi|+v|\phi|
\end{aligned}
$$

In the expressions, $\phi$ is the level set function, $\omega(0 \leq \omega \leq 1)$ is the weight parameters, $\mu, v \geq 0, F$ is the clustering coefficient of the template. When dealing with Micro-CT medical slice images, the size of the image and the size of the clustering template are equal, then, according to the clustering, as defined by the template image in the target location area part is 1 , the background area part is 0 , the initial level set to obtain an image based on the level set in the initial curve clustering information, however, opposed to the previous rules of the initial curve, K-LGIF model not only effectively enhanced the Micro-CT medical image which contrast lower part of segmentation, but also improved the image of the target portion of the divided recognition efficiency.

In Eq.(1), $H_{\varepsilon}(\phi)$ is regularized Heaviside function, the expression is:

$$
\begin{aligned}
& H_{\varepsilon}(\phi)=\frac{1}{2}\left[1+\frac{2}{\pi} \arctan \left(\frac{\phi}{\varepsilon}\right)\right] \\
& \delta_{\varepsilon}(\phi)=H_{\varepsilon}^{\prime}(\phi)=\frac{1}{\pi} \frac{\varepsilon}{\varepsilon^{2}+\phi^{2}}
\end{aligned}
$$

We can use Euler-Lagrange equation:

$$
\int K_{\sigma}(x-y)\left(I(y)-f_{1}(x)\right) H_{\varepsilon}(\phi(y)) d y=0
$$




$$
\begin{aligned}
& \int K_{\sigma}(x-y)\left(I(y)-f_{2}(x)\right)\left(1-H_{\varepsilon}(\phi(y))\right) d y=0 \\
& \int F .\left(I(x)-c_{1}\right) H_{\varepsilon}(\phi(x)) d x=0 \\
& \int F .\left(I(x)-c_{2}\right)\left(1-H_{\varepsilon}(\phi(x))\right) d x=0
\end{aligned}
$$

Further available:

$$
\begin{aligned}
& f_{1}=\frac{K_{\sigma}(x) *\left[H_{\varepsilon}(\phi(x)) I(x)\right]}{K_{\sigma}(x)^{*} H_{\varepsilon}(\phi(x))} \\
& f_{2}=\frac{K_{\sigma}(x) *\left[\left(1-H_{\varepsilon}(\phi(x))\right) I(x)\right]}{K_{\sigma}(x) *\left(1-H_{\varepsilon}(\phi(x))\right)} \\
& c_{1}=\frac{\int F \cdot I(x) H_{\varepsilon}(\phi(x)) d x}{\int H_{\varepsilon}(\phi(x)) d x} \\
& c_{2}=\frac{\int F \cdot I(x)\left(1-H_{\varepsilon}(\phi(x))\right) d x}{\int\left(1-H_{\varepsilon}(\phi(x))\right) d x}
\end{aligned}
$$

Where $f_{1}, f_{2}$ are all local capacity in the function, and they are the weighted average of the gray areas within the local scope point $\mathrm{x}$ in the image, so for the intensity distribution of non-uniform image segmentation, the local binary fitting model has good segmentation effect. $c_{1}, c_{2}$ all of the energy functional in order to add clustering information in the energy functional in Eq.(10) and Eq.(11), to obtain the global optimal image segmentation results. The accession of clustering information made the segmentation speed improved. When the image over-segmentation phenomenon, the value of $\omega$ should take a larger value to make LGIF model tended to GIF model to segment images. When the gray background of the target image is not uniform, $\omega$ should take a smaller value to make LGIF model tended to LIF model to segment images.

\section{Analysis of geometric characteristics of mice trabecular bone}

Through the binary image of the trabecular bone, we can calculate the following three geometric shape factors[9-10]: mean trabecular plate thickness (MTPT), trabecular bone volume (TBV), and trabecular bone number $(N)$. This description can be used for quantitative analysis of bone shape measurement.

When the occurrence of osteoporosis, mean trabecular plate thickness rabecular bone volume, number of parameters will change, when calculating the average mouse femoral bone trabecular thickness, we can see section in the slice of trabecular bone image approximately treated as an irregular ring. Mean trabecular plate thickness refers to the average thickness of the slice of trabecular bone, when calculating the thickness of ring, you can set $D$ as its outer diameter and $d$ as inner diameter, then, $D$ - $d$ indicates the thickness of the ring. When using the area $(S)$ of ring to divide the sum of inner and outer circumference $(C)$, we can get:

$$
\frac{S}{C}=\frac{\pi D^{2}-\pi d^{2}}{2 \pi D+2 \pi d}=\frac{D-d}{2}
$$


Mean trabecular plate thickness can seen as: $M T P T=2 * \frac{S}{C}$, then use the captured image and the kind of scale, you can calculate the approximate value of the actual mean trabecular plate thickness.

The trabecular bone volume refers to the section of the trabecular bone volume percentage of all marrow cavity, the marrow cavity and bone trabecular in the slice approximately as a cylinder, the volume computation formula of mouse femoral trabecular bone is as follows, which $V_{\text {trabecular bone }}$ refers to the measured bone trabecular area, $V_{\text {all }}$ refers to the measured the femoral area:

$$
T B V=\frac{V_{\text {trabecular bone }}}{V_{\text {all }}}
$$

Trabecular bone number (n) is another factor to describe the shape feature of trabecular bone, calculating the number of trabecular bone and mark the centroid based on the thought of connected domain in the mark images, which can be quickly and effectively to achieve the number of trabecular bone.

\section{Experimental Results and Analysis}

\section{Time Consuming Experiments of Micro-CT Slice Images of the Mouse Femur}

The environmental simulation test of this experiment under Windows 7 which RAM is $4.00 \mathrm{~GB}$, MATLAB R2012b. In the test, $\lambda_{1}=1, \lambda_{2}=1$, Time step $\Delta t=0.1, \mu=1$, $\varepsilon=3.0, \omega=0.01$. CV model is not able to separate the target completely separate from the background based on Micro-CT slice image of mouse femur which characteristic is low contrast and the image size is $764 \times 764$, and our model is divided relatively CV model improves 12.48 times, LBF model for non-uniform gray image segmentation effect is better than CV model, but it is not better than LGIF model in contrast slightly lower part. The running time of K-LGIF is better than LGIF model, the speed of segmentation with K-LGIF model than LGIF model improved 0.56 times. The experimental results are shown in Fig. 1 and Table 1.

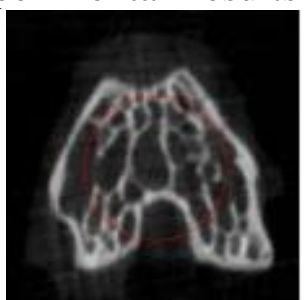

(1)

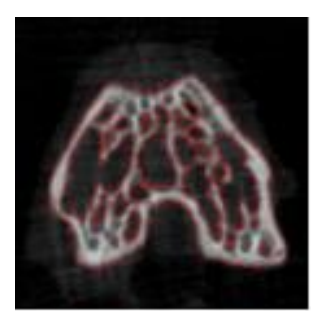

(1)

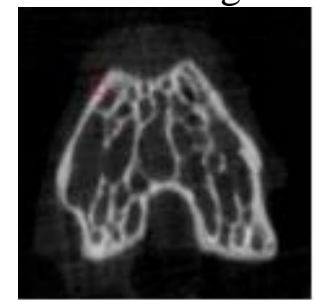

(2)

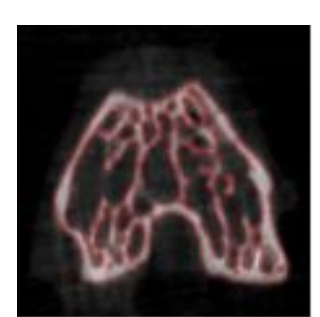

(2)

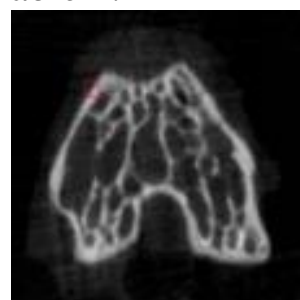

(3)

(a)

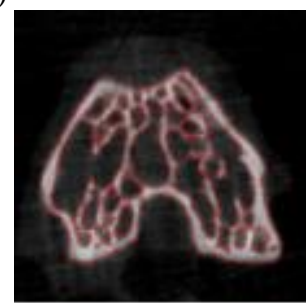

(3)

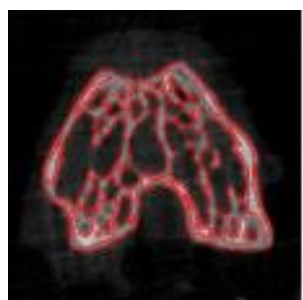

(4)

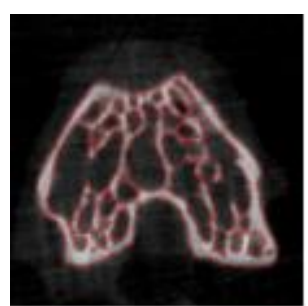

(4)

(b)

Fig. 1. Micro-CT slice images of the mouse femur (1)the initial contour of CV model (2)the initial contour of LBF model (3)the initial contour of LGIF model (4)the initial contour of K-LGIF model in (a), (1)the result of segmenting CV model (2)the result of segmenting LBF model (3)the result of segmenting LGIF 
model (4)the result of segmenting K-LGIF model in (b).

Table 1. Results of segmenting dates comparison on images of Micro-CT in different models

\begin{tabular}{ccccc}
\hline The information of image & CV model & $\begin{array}{c}\text { LBF } \\
\text { model }\end{array}$ & $\begin{array}{c}\text { LGIF } \\
\text { model }\end{array}$ & K-LGIF model \\
\hline Number of iterations [times] & 4000 & 200 & 80 & 50 \\
Running of time[s] & 323.10 & 90.68 & 37.36 & 23.97 \\
Relative efficiency with & 12.48 & 2.78 & 0.56 & 0.00 \\
K-LGIF model[multiple] & & & \\
\hline
\end{tabular}

The Experimental Analysis of Geometric Characteristics on the Trabecular Bone of Mouse Distal Femoral

According to the above parameters are calculated statistics which is the number of trabecular bone, mean trabecular plate thickness and trabecular bone volume. An experiments of image is selected from Fig. 1 which are shown in Fig. 2 and a random slice images of mouse femur, the number of statistical structure of trabecular bone and mark the center of mass.

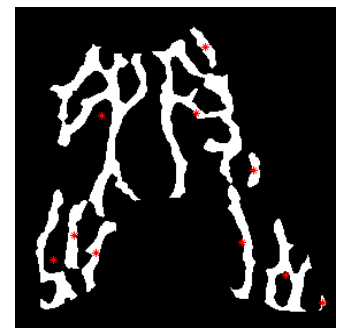

(a) trabecular bone I

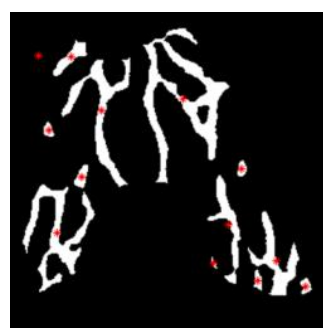

(b)trabecular bone II

Fig. 2. Number of trabecular bone of mouse distal femur

Table 2 shows the mean trabecular plate thickness and trabecular bone volume of the slice image. The average error of mean trabecular plate thickness and trabecular bone volume on trabecular bone I, II was, respectively, $0.40 \%, 0.06 \%, 4.31 \%, 1.57 \%$, this way can effectively Statistic geometric features which is mean trabecular plate thickness and trabecular bone volume.

Table 2. Geometric features of the average of a number of slices and the calculated values of trabecular bone in mouse distal femur

\begin{tabular}{cccc}
\hline $\begin{array}{c}\text { Sample } \\
\text { Calculation }\end{array}$ & Sample name & $\begin{array}{c}\text { Mean trabecular plate } \\
\text { thickness[ }[\mu \mathrm{m}]\end{array}$ & $\begin{array}{c}\text { Trabecular bone } \\
\text { volume }(\%)\end{array}$ \\
\hline \multirow{2}{*}{ Mean of samples } & trabecular bone I & 67.30 & 16.01 \\
& trabecular bone II & 67.55 & 15.93 \\
Single slice value & trabecular bone I & $67.57(+0.40 \%)$ & $16.70(+4.31 \%)$ \\
& trabecular bone II & $67.59(+0.06 \%)$ & $15.68(-1.57 \%)$ \\
\hline
\end{tabular}

\section{Conclusions}

This paper overcomes the shortcomings of the initial contours as the standard geometry with CV model, LBF model, LGIF model in this paper, The K-LGIF model combined with the advantages of $\mathrm{CV}$ model and LBF model form LIGF model to segment effectively gray uneven medical slice images of Micro-CT, The clustering template is added in LGIF model can identify the preliminary identification of target contour in the image to improve the speed of running. The K-LGIF model for Micro-CT medical images segmentation efficiency relative to CV model and LBF model and LGIF model respectively increased an average of 12.48 times, 2.78 times, 0.56 times. Through the 
simulation with a mouse, a period of time, the same parts of the occurrence osteoporosis bone which the trabecular bone changed thinning, loss and fracture in the experiment can be seen that the parameters of trabecular plate thickness and bone volume changed significantly from the parameters of gray level co-occurrence matrix, the above method can provide help for the fast segmentation of medical image on Micro-CT and doctor's diagnosis, in which the parameter variations can be as a research method of the study on osteoporosis.

\section{Acknowledgement}

This research was financially supported by the Industry-Academia-Research Joint Innovation Fund of Jiangsu Province (BY2014037-11).

\section{References}

[1] Z. Peter, V. Bousson, C. Bergot, Pattern Recognition, 41( 2008) 2358-2368.

[2] Satoshi Sashida, Yutaka Okabe, Hwee Kuan Lee, Computer Vision and Image Understanding, 119 (2014) 15-26.

[3] Qin Zhang, Dewen Hou, Computer Applications and Software, 31 (2014) 214-216.In Chinese

[4] Chunming Li, ChenyangXu, Distance Regularized LevelSet Evolution and Its Application to Image Segmentation[C]. IEEE Trans On Image Processing, 19 (2010) 590-622.

[5] Yan Zhou, Wei-Ren Shi, Wei Chen, Neurocomputing, 156 ( 2015) 199-210.

[6] Chunming Li, Chiu-Yen, Kao, JohnC. Gore, Implicit Active Contours Driven by Local Binary Fitting Energy[C]. The Computer Vision and Pattern Recognition, CVPR. 07. $\operatorname{IEEE}(2007)$.

[7] Min Li, Jiuzhen Liang, Cuicui Liao, PR \& AI, 28 (2015) 665-672.(In Chinese)

[8] Deep Gupta, R.S. Anand, Barjeev Tyagi, Biomedical Signal Processing and Control, 16 (2015) 98-112.

[9] Guoding Shen, The parameters of bone histomorphometry can be automatically ext racted and applied base on trabecular bone of Micro-CT[D]. TONGJI University, 2001. (In Chinese)

[10] Narihiro Okazaki, Ko Chiba, Kenji Taguchi, et al, Bone, 03 (2014) 82-87. 